\title{
ROSAT Deep Surveys
}

\author{
Günther Hasinger \\ Max-Planck-Institut für Extraterrestrische Physik \\ 8046 Garching, Germany
}

\begin{abstract}
A series of 20 deep pointed observations with the ROSAT PSPC is discussed. 530 X-ray sources

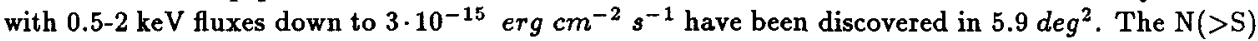
relation of the sources selected in the $0.5-2 \mathrm{keV}$ band shows a density in excess of $200 \mathrm{deg}^{-2}$ at

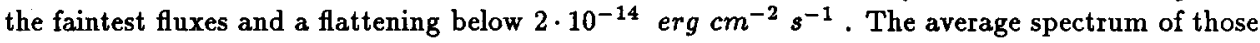
sources is a power law with energy index $1.2 \pm 0.1$. The absorption column densities are consistent with the galactic HI columns. More than $50 \%$ of the 1-2 $\mathrm{keV}$ background has been resolved into discrete sources in the deepest field. The total background spectrum shows an emission line feature around $0.65 \mathrm{keV}$, most probably due to OVII-OVIII from a $2 \cdot 10^{6} \mathrm{~K}$ plasma. Above $\sim 1 \mathrm{keV}$ the background is dominated by a power law spectrum with a normalization of $13.4 \pm 0.2$

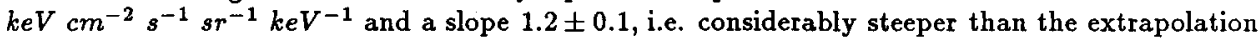
from higher energies.
\end{abstract}

\section{Introduction}

Deep imaging studies of the X-ray background have first been performed with the Einstein IPC and HRI (Giacconi et al., 1979, Griffiths et al., 1983, Primini et al., 1991). These observations were able to resolve about $20 \%$ of the background at $2 \mathrm{keV}$ into sources, the majority of which turned out to be extragalactic. The PSPC (Pfeffermann et al., 1986) aboard ROSAT (Trümper, 1983) comprises a few features which make it particularly useful for the study of the soft X-ray background: its extremely low intrinsic background (Snowden et al., 1991), its good energy resolution, temporal gain stability and spatial homogeneity. In addition, the large collecting area and large field of view of the ROSAT telescope are particularly useful. Consequently, every pointed PSPC observation contains a number of serendipitous sources. About half of all PSPC pointings reach sensitivity levels comparable to or fainter than the Einstein Deep Surveys.

First results on medium-deep pointed observations have already been published elsewhere (Hasinger et al., 1991, hereafter paper I; Shanks et al., 1991). Here I report on further results from early long PSPC observations at high galactic latitudes (RMSS) and on the recent ROSAT Deep Survey, obtained in the Lockman Hole, a region with the absolutely lowest neutral hydrogen interstellar column density (Lockman et al., 1986). This project is performed in collaboration with R.Burg, R.Giacconi (both STScI), G.Hartner, J.Trümper (both MPE), M.Schmidt (Caltech) and G.Zamorani (Bologna). Section 2 gives an overview of the selection of survey fields. In section 3 
the $\mathrm{X}$-ray $\log \mathrm{N}-\log \mathrm{S}$ relation is derived. Section 4 and 5 describe analytic fits to the spectra of $\mathrm{X}$-ray sources and the background, repectively.

\section{Field Selection}

Table 1 summarizes the selected survey fields, all at high galactic latitudes and with exposure times greater than $8 \mathrm{ksec}$. Limiting fluxes in the $0.5-2 \mathrm{keV}$ band have been calculated assuming a power law spectrum with energy index of -1 . Fields marked with an asterisk have been included in the analysis by courtesy of the principal investigators owning the data rights. Analysis of eight of the current fields has been published in paper I. A more thorough report on the Lockman Hole data is in preparation, as is a detailed description of the fits to the $\mathrm{X}$-ray background spectrum.

Table 1: Field Selection

\begin{tabular}{|c|c|c|c|c|c|c|c|c|}
\hline $\begin{array}{l}\text { Field } \\
\text { Name }\end{array}$ & $\begin{array}{l}\text { Gal. } \\
\text { Long. } \\
{[d e g]}\end{array}$ & $\begin{array}{l}\text { Gal. } \\
\text { Lat. } \\
{[d e g]}\end{array}$ & $\begin{array}{l}\text { Column } \\
\text { Density } \\
{\left[\mathrm{cm}^{-2}\right]}\end{array}$ & $\begin{array}{l}\text { Exp. } \\
\text { Time } \\
{[s]}\end{array}$ & $\begin{array}{c}\text { "Clean" } \\
\text { Part } \\
{[\%]}\end{array}$ & $\begin{array}{l}\text { Nr. } \\
\text { Src. }\end{array}$ & $\begin{array}{c}S_{\min } \\
(0.5-2 \mathrm{keV}) \\
{\left[\operatorname{erg~} \mathrm{cm}^{-2} \mathrm{~s}^{-1}\right]}\end{array}$ & $\begin{array}{c}\text { Fraction } \\
\text { Resolved } \\
{[\%]}\end{array}$ \\
\hline$\alpha$ Boo*,+ & 15 & 69 & $2.5 \cdot 10^{20}$ & 17100 & - & 31 & $0.93 \cdot 10^{-14}$ & 34.8 \\
\hline$\beta \mathrm{Leo}^{+}$ & 251 & 71 & $3.1 \cdot 10^{20}$ & 20160 & 31 & 25 & $0.76 \cdot 10^{-14}$ & 34.5 \\
\hline$\delta$ Leo & 224 & 67 & $1.1 \cdot 10^{20}$ & 21630 & - & 37 & $0.83 \cdot 10^{-14}$ & 42.6 \\
\hline EF Eri' ${ }^{*++}$ & 214 & -58 & $1.8 \cdot 10^{20}$ & 15840 & - & 23 & $0.87 \cdot 10^{-14}$ & 28.7 \\
\hline EX Hya* & 303 & 34 & $6.1 \cdot 10^{20}$ & 15620 & 32 & 16 & $1.44 \cdot 10^{-14}$ & 22.9 \\
\hline HR $857^{*,+}$ & 192 & -58 & $3.2 \cdot 10^{20}$ & 10680 & - & 7 & $1.16 \cdot 10^{-14}$ & 31.2 \\
\hline $\mathrm{HZ} 43^{+}$ & 54 & 84 & $1.0 \cdot 10^{20}$ & 13100 & 3 & 32 & $0.70 \cdot 10^{-14}$ & 44.6 \\
\hline $\mathrm{HZ} 43 \mathrm{P}$ & 54 & 84 & $1.0 \cdot 10^{20}$ & 22530 & 41 & & & \\
\hline LHS $2924^{*}$ & 55 & 68 & $1.1 \cdot 10^{20}$ & 10680 & 21 & 27 & $0.51 \cdot 10^{-14}$ & 32.3 \\
\hline Lockman & 149 & 53 & $0.6 \cdot 10^{20}$ & 78320 & 23 & 92 & $0.28 \cdot 10^{-14}$ & 50.7 \\
\hline $\operatorname{Lynx} x^{*}$ & 176 & 53 & $2.7 \cdot 10^{20}$ & 66900 & 34 & & & \\
\hline Meaty & 111 & 53 & $4.0 \cdot 10^{20}$ & 23400 & - & 21 & $0.60 \cdot 10^{-14}$ & 47.0 \\
\hline $\mathrm{NEP}^{+}$ & 96 & 30 & $3.9 \cdot 10^{20}$ & 49200 & 11 & 41 & $0.44 \cdot 10^{-14}$ & 37.8 \\
\hline NEPN & 96 & 30 & $3.9 \cdot 10^{20}$ & 41760 & - & 44 & $0.62 \cdot 10^{-14}$ & 43.0 \\
\hline Nower 1 & 202 & 55 & $1.8 \cdot 10^{20}$ & 41760 & 18 & 40 & $0.44 \cdot 10^{-14}$ & 34.3 \\
\hline Nower2 & 136 & 68 & $1.1 \cdot 10^{20}$ & 30590 & 10 & 38 & $0.57 \cdot 10^{-14}$ & 29.2 \\
\hline Pavo* & 319 & -77 & $5.3 \cdot 10^{20}$ & 25080 & - & 29 & $0.99 \cdot 10^{-14}$ & 32.0 \\
\hline $\mathrm{SEP}^{+}$ & 276 & -30 & $5.3 \cdot 10^{20}$ & 20220 & 8 & 16 & $0.79 \cdot 10^{-14}$ & 34.8 \\
\hline VW Hyi $^{*},+$ & 285 & -38 & $8.5 \cdot 10^{20}$ & 8760 & 27 & 11 & $1.47 \cdot 10^{-14}$ & 32.6 \\
\hline Warlock* & 161 & 53 & $0.7 \cdot 10^{20}$ & 34860 & 53 & & & \\
\hline
\end{tabular}

$$
\text { * Courtesy AO-1 PIs + paper I }
$$

Figure 1 shows the image of the Lockman Field in the 0.1-2.4 keV band. The data have been background-subtracted and smoothed with a Gaussian with a FWHM of 28". About 90 discrete $\mathrm{X}$-ray sources have been detected in the inner, most sensitive part of the field of view, marked by the circular shadow of the PSPC window support structure at a radius of 20 arcmin.

\section{3. $\log N-\log S$ Function}

The source detection process has been described in some detail in paper I. The same sliding box algorithm has been applied here, however separately for 4 energy bands: S (0.1-0.4 keV), H 


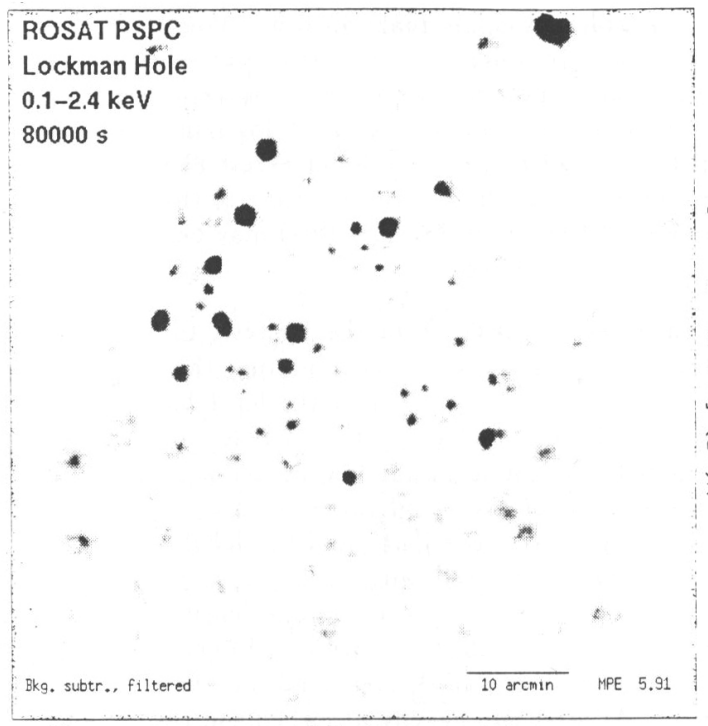

Figure 1: X-ray Image of the Lockman Hole

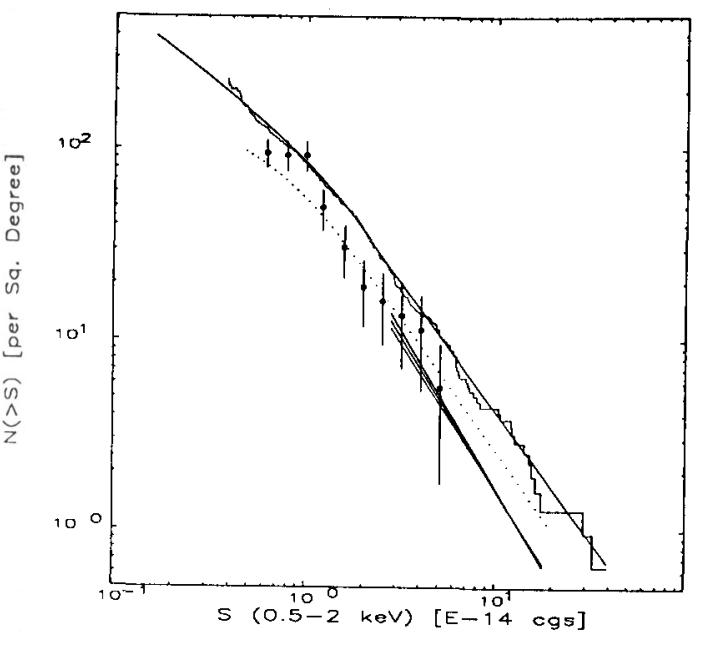

Figure 2: ROSAT $\log N-\log S$ compared to EMSS

(0.4-2.4 keV), T (0.1-2.4 keV) and J (0.9-2.4 keV). In the previous analysis source counts were accumulated in a ring of radius 40 " around each source. In the meantime more calibration data became available, and it turned out that the above radius was too small, in particular at larger off-axis angles and that the source fluxes have been systematically underestimated by $30 \%$ on the average. For the current flux and significance determination a $90 \%$ radius has been determined separately for each source as a function of off-axis angle and average energy band (eg. between 29 " and 69 " in the H-band). The source detection threshold was set at a likelihood of $L=-\ln (P)=10$, corresponding to not more than a couple of spurious sources in the whole survey. A total of 530 discrete sources was detected in the inner $20^{\prime}$ of 17 fields $\left(5.9 \mathrm{deg}^{2}\right)$ in at least one of the above energy bands. Source count rates were converted to incident fluxes in the $0.5-2 \mathrm{keV}$ band assuming a power law spectrum with energy index -1 and galactic $H_{I}$ absorption.

For the construction of a preliminary $\log N-\log S$ function the sample has been restricted to detections and fluxes in the H-band and at off-axis angles less than 15'. A sensitivity histogram (surveyed area as a function of limiting flux) was constructed taking into account the variing sensitivity as a function of off-axis angle. 317 sources in the flux interval $3 \cdot 10^{-15}-4 \cdot 10^{-13}$ $e r g \mathrm{~cm}^{-2} \mathrm{~s}^{-1}$ remained in the sample. In figure 2 the integral $N(>S)$ function derived in this work (histogram) is displayed together with the estimate from paper $I$ (dotted line). Due to the above $30 \%$ systematic flux error, the two curves are shifted with respect to each other. A broken power law with the two slopes $\alpha_{1}, \alpha_{2}$ and a break flux $S_{B}$ was fit to the differential source counts. The solid line shows the integrated best fit function with the parameters $\alpha_{1}=2.4 \pm 0.2, \alpha_{2}=1.7 \pm 0.3$, $S_{B}=2.2 \pm 0.9 S_{14}(90 \%$ errors $)$ and normalization of 89.6 at $S_{14}=10^{-14} \mathrm{erg} \mathrm{cm}^{-2} \mathrm{~s}^{-1}$. The flattening of the source counts, as already indicated in paper $I$ could be confirmed here, however, it appears less dramatic. An extrapolation of the $\log N-\log S$ function to fainter fluxes can account for $80 \pm 20 \%$ of the total 1-2 keV background.

Because spectroscopic identifications are not yet available the dataset contains galactic as well as extragalactic objects. However, preliminary X-ray/optical correlations and the first spectroscopic identifications (Shanks et al., 1991) indicate that the stellar content is small $(\sim 10 \%)$, compared to about $25 \%$ in the EMSS (Gioia et al., 1990). Figure 2 also contains the source counts 
of the first spectroscopically identified ROSAT field (Shanks et al., 1991; filled dots) and the fit to the EMSS AGN (Gioia et al., 1990, straight solid lines). In order to convert the EMSS fluxes (0.3$3.5 \mathrm{keV}$ ) to our $0.5-2 \mathrm{keV}$ passband a power law source spectrum with energy index -1 has been assumed. The mismatch between these two datasets and the new ROSAT $\log N-\operatorname{logS}$, in particular at bright fluxes may be partially due to the stellar content and due to the fact that Shanks et al. (1991) did not add the contribution of fluxes brighter than $6 \cdot 10^{-14} \mathrm{erg} \mathrm{cm}^{-2} \mathrm{~s}^{-1}$ to their counts. Also a systematic flux uncertainty between the EMSS and the RMSS (20-30\%) may be present.

\section{Average Spectra of Resolved Sources}

Sources brighter than $10^{-13} \mathrm{erg} \mathrm{cm}^{-2} \mathrm{~s}^{-1}$ have been omitted from the analysis to minimize statistical fluctuations. Average source spectra were determined by accumulating the counts in rings containing more than $95 \%$ of the photons of all sources detected in the hard band in the inner region (15' radius) of each field. The average background (sky plus particle), determined from the remaining adjacent pixels, was subtracted. A simple power law model with cold gas absorption could be fit to the average source spectrum of every individual field in the energy range 0.1-2 keV. Figure 3 compares the fits to the spectra of the HZ43 and the NEP field. The power law slopes derived in the individual fields agree very well with each other, yielding an average value of $\alpha_{E}=1.16 \pm 0.08$. The derived values of the absorption column densities $N_{H}$ are consistent with the values derived from 21-cm maps of the galactic neutral lydrogen. Spectral fits to individual sources in general agree very well with this model, in a small number of cases, however, significantly different spectra are present. At least two heavily absorbed sources and one object with a comparatively soft spectrum have been found in the Lockman hole.

The integral flux of the average source spectra directly yields the contribution of sources with fluxes between the sensitivity limit of the corresponding field and the upper flux limit $\left(10^{-13}\right.$ erg $\mathrm{cm}^{-2} \mathrm{~s}^{-1}$ ) to the total background in the $1-2 \mathrm{keV}$ band. In order to quantify the total amount of background already resolved into sources a correction for the contribution of sources brighter than the upper flux threshold has to be applied. This was derived from an extrapolation of the above $\mathrm{N}(>\mathrm{S})$ relation to the bright end. In table 1 and in figure 4 the fraction of background resolved in the $1-2 \mathrm{keV}$ band is given. In the Lockman field the total resolved flux corresponds to a power law normalization of $6.8 \pm 0.3 \mathrm{keV} \mathrm{cm}^{-2} \mathrm{~s}^{-1} \mathrm{sr}^{-1} \mathrm{keV}^{-1}$ at $1 \mathrm{keV}$.

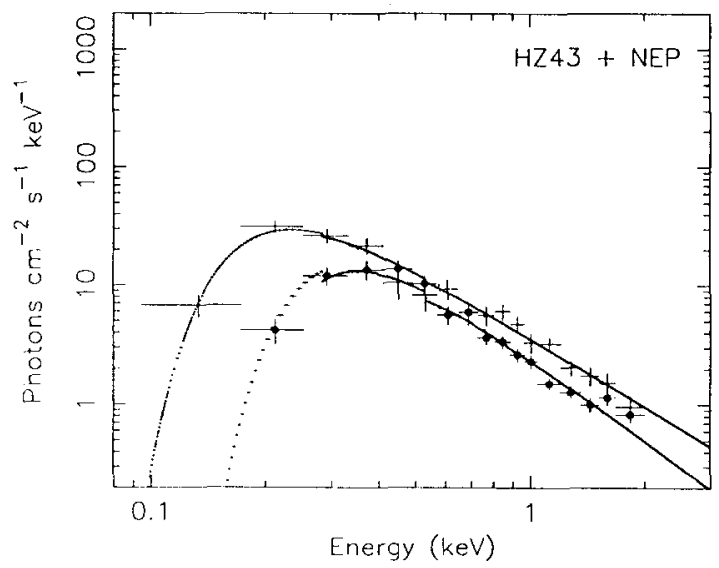

Figure 3: Average source spectra

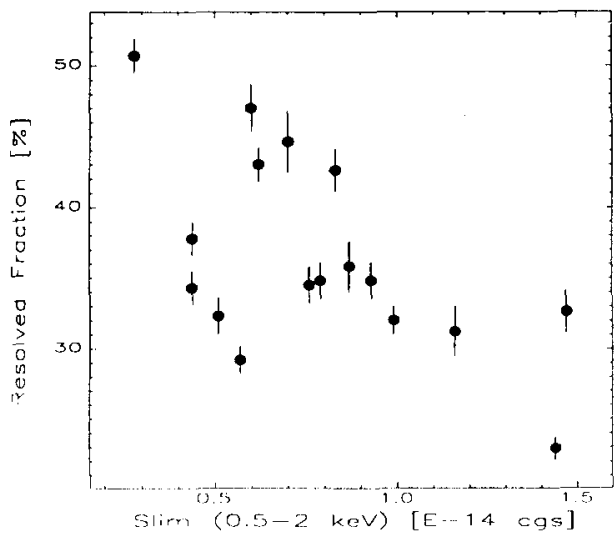

Figure 4: Resolved fraction 


\section{The Cosmic X-ray Background Spectrum}

To minimize the contamination by non-cosmic background components, mainly the particle background, which is strongest at high geomagnetic latitudes (Snowden et al., 1991), and the solar scattered light plus a geocoronal oxygen line at $0.53 \mathrm{keV}$, which both can be very strong on the dayside of the satellite orbit (see eg. Fink et al., 1988), restrictive selection criteria have been applied. Only observation intervals at geographic latitudes between -30 and 30 degrees (tropic) and with Sun-Earth-satellite angles larger than 120 degrees (night) have been accepted. On average the tropic night data comprises about $25 \%$ of the net observing time, but there are quite a few observations without useful clean observation periods (see table 1).

To improve on the statistical quality of the data background spectra have generally been accumulated over the whole PSPC field- of-view ( $0-57$ ' radius), however, if a bright target (with broad scattering wings) was observed, or if the boron filter (covering a central circle with radius $\sim 25^{\prime}$ ) was used, the analysis was restricted to the outer field-of-view (27'-57').

The particle background with the spectral shape given by Snowden et al. (1991) was subtracted, normalized to the highest energy channels $(2.4-2.7 \mathrm{keV})$, where the sky contribution can be neglected. Some fields indicate a further, slowly time variable background component with a relatively soft spectrum which is as yet not understood. Affected times were excluded as far as possible from the analysis. Fields on or close to the north-polar spur have also been discarded.

First a relatively simple spectral fit was attempted with a $10^{6} \mathrm{~K}$ thermal plasma for the carbon-band (0.1-0.3 keV) emission (see McCammon and Sanders, 1990) and a single power law with fixed galactic absorption for the higher energies. Figure 5 shows the residuals to this fit for the HZ43P field, which display a significant excess in the $0.5-0.7 \mathrm{keV}$ range, resembling an emission line with a centroid energy of $\sim 0.65 \mathrm{keV}$, i.e. higher than that of a monochromatic oxygen $K \alpha$ line, and therefore most probably due to ionized oxygen (OVII-OVIII). This line, which is present in practically all fields, confirms the existence of hot plasma at temperatures of $2-3 \cdot 10^{6} \mathrm{~K}$ (Inoue et al., 1980; Rocchia et al., 1984), distributed over the whole sky.

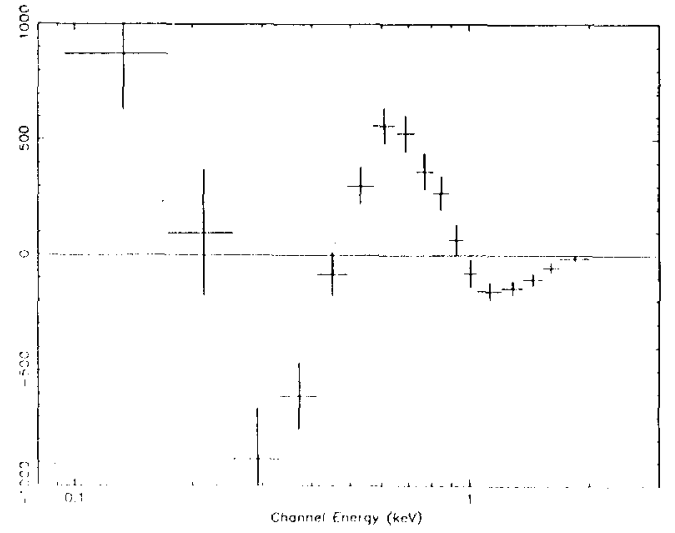

Figure 5: Power law residuals

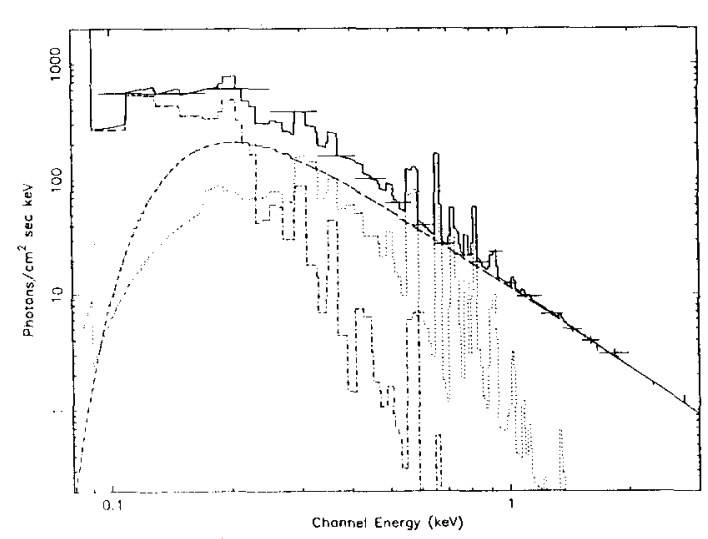

Figure 6: Composite Background Fit

Therefore, a more complicated spectrum had to be fit, with two thermal line spectra at one million and 2-3 million degrees, respectively, plus a power law absorbed through the galaxy for the higher energy extragalactic component. The best-fit absorption for the hot thermal component is strongly correlated with the flux of the soft thermal component and therefore was fixed at a rather arbitrary value of $5 \cdot 10^{19} \mathrm{~cm}^{-2}$. This fit yielded acceptable $\chi^{2}$ values for all analysed fields. The best-fit values of the power law index and flux, as well as the temperature and flux of the hot thermal component are consistent with being constant in all fields, while the flux of the soft 
thermal component varies strongly over the sky. The following average values have been derived for the different parameters: power law energy index $\alpha_{E}=1.12 \pm 0.07$, power law norm $13.4 \pm 0.3$ $\mathrm{keV} \mathrm{cm}^{-2} \mathrm{~s}^{-1} \mathrm{sr}^{-1} \mathrm{keV}^{-1}$ (at $1 \mathrm{keV}$ ), temperature of hot component $0.20 \pm 0.01 \mathrm{keV}$.

Figure 7 (adapted from McCammon and Sanders 1990) shows a comparison of the ROSAT background spectrum (power law component only; two crossing solid lines) with the Wisconsin data and the the extrapolation of the HEAO-1 spectrum (lower dashed line; Marshall et al., 1980). The power law, dominating above $1 \mathrm{keV}$, meets the HEAO-1 extrapolation at roughly 2 $\mathrm{keV}$, but is considerably steeper. The ROSAT flux density at $1 \mathrm{keV}$ is about $10 \%$ lower than that of the Wisconsin survey, consistent with the uncertainties in the relative calibration of the two instruments. The figure also contains the average spectrum of the resolved sources with the normalization taken from the Lockman Hole data (long dashes); the two arrows indicate that this is a lower limit of the total source contribution to the X-ray background.

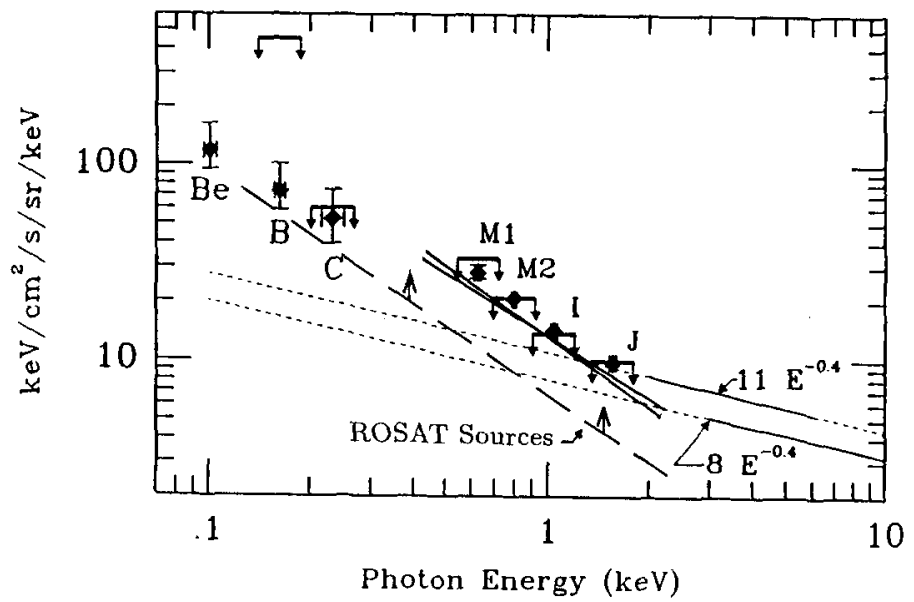

Figure 7: Comparison between Wisconsin and ROSAT background spectrum

\section{References}

Fink H.H., Schmitt J.H.M.M. \& Harnden F.R.,Jr. Astr. Astrophys. 193, 345 (1988).

Giacconi R. et al. Astrophys. J. 234, L1 (1979).

Gioia I. M. et al. Astrophys. J. suppl. Ser. 72, 567 (1990).

Griffiths R.E. et al. Astrophys. J, 269, 375 (1983).

Hasinger G., Schmidt M. \& Trümper J. Astr. Astrophys. 246, L2 (1991) (paper I).

Inoue H. et al. Astrophys. J. 238, 886 (1980).

Lockman F.J., Jahoda K. \& McCammon D. Astrophys. J. 302, 432 (1986).

Marshall F.E. et al. Astrophys. J. 235, 4 (1980).

McCammon D. \& Sanders W.T. Ann. Rev. Astron. Astrophys. 28, 657 (1990).

Primini F.A. et al. Astrophys. J. 374, 440

Pfeffermann E. et al. Proc. SPIE 733, 519 (1986).

Rocchia, R. et al. Astr.Astrophys. 130, 53 (1984).

Snowden S. et al., Astrophys.J. (submitted) (1991).

Shanks T. et al., Nature 253, 315 (1991).

Trümper J. Adv. Space Res. 2, no.4, 241 (1983).

A cknowledgements: I am grateful to the ROSAT team, my collaborators and various AO-1 PIs. 